\title{
Otimização do processo de dispersão de nanotubos de carbono em poliuretano termorrígido
}

\section{Optimization of carbon nanotubes dispersion process in thermoset polyurethane}

\author{
Magnovaldo Carvalho Lopes', João Paulo Campos Trigueiro', Vinicius Gomide de Castro', \\ Rodrigo Lassarote Lavall ${ }^{1}$ and Glaura Goulart Silva ${ }^{1 *}$

\begin{abstract}
'Laboratório de Materiais Poliméricos Multicomponentes, Departamento de Química, Universidade Federal de Minas Gerais - UFMG, Minas Gerais, MG, Brasil

glaura.goulart@gmail.com
\end{abstract}

\begin{abstract}
Resumo
Neste trabalho foi desenvolvido um processo empregando misturador de alto cisalhamento e moinho de rolos para dispersar MWCNTs (multiwalled carbon nanotubes) puros e modificados em poliol visando a preparação de concentrados de $3 \%$ em massa. Condições otimizadas no trabalho permitiram a obtenção de suspensões com menor número e tamanho de agregados de MWCNTs. Compósitos contendo 0,5\% em massa de MWCNTs foram preparados por diluição dos concentrados em poliol usando mistura mecânica seguida de cura. Resultados de microscopia indicaram que as melhores dispersões foram obtidas com os MWCNTs modificados, os quais permitiram um aumento na tensão na ruptura, no alongamento e uma melhor preservação da estabilidade térmica. Além disso, valores de condutividade elétrica sugerem que o compósito possa ser empregado para dissipação eletrostática. Dessa forma, os resultados obtidos demonstram que a modificação covalente da superfície dos MWCNTs e a utilização de estratégias eficientes de dispersão são essenciais para melhorar as propriedades finais dos nanocompósitos.
\end{abstract}

Palavras-chave: dispersão de nanotubos de carbono, nanotubos de carbono modificados, propriedades mecânicas, poliuretano termorrígido elastomérico.

\begin{abstract}
A process employing high shear mixer and roll mill to disperse pristine and modified MWCNTs (multiwalled carbon nanotubes) in polyol was developed in order to prepare $3 \mathrm{wt} \%$ masterbatches. The optimum process conditions resulted in suspensions with smaller number and size of nanotube aggregates. Composites containing $0.5 \mathrm{wt} \%$ of MWCNTs were prepared by dilution of polyol masterbatches by simple mechanical mixing followed by cure. Microscopy data revealed better dispersion of modified carbon nanotubes in the polymer matrix, which promoted an increase in the tensile strength, elongation and a better preservation of thermal stability. Furthermore, electric conductivity values indicated that the composites can be used for electrostatic dissipation. These results demonstrate that the covalent modification of MWCNTs surface and the use of efficient dispersion strategies are essential to improve nanocomposites' final properties.
\end{abstract}

Keywords: carbon nanotubes dispersion, modified carbon nanotubes, thermoset polyurethane elastomer, mechanical properties.

\section{Introdução}

A primeira descrição relacionada ao processo de produção de poliuretanos (PUs) baseado na reação de um diisocianato com um diol foi a patente alemã depositada pela I.G. Farben (subdivisão da Bayer) em 1937, tendo Otto Bayer como um dos inventores. ${ }^{[1]}$

As propriedades dos PUs e portanto sua aplicação final, dependem do isocianato e diol empregados (e da proporção entre eles), além de outros reagentes como extensores de cadeia, agentes de cura, aditivos, entre outros, bem como do processo envolvido na sua síntese. Esse processo pode acontecer em uma etapa (one shot), no qual todos os

reagentes são adicionados no início da reação ou em duas etapas, via produção de um pré-polímero (reação entre o isocianato e poliol) que é posteriormente reagido com o extensor de cadeia ou agente de cura (sistemas com um ou dois componentes $)^{[1,2]}$. Podem ser produzidos poliuretanos termoplásticos ou termorrígidos, preparando-se desde espumas rígidas ou flexíveis, passando por revestimentos resistentes a produtos químicos, adesivos especiais, selantes, pequenos componentes de máquinas como engrenagens, rodízios e roldanas, até grandes peças industriais de alto desempenho mecânico ${ }^{[1,2,3]}$. 
A presença de ligações covalentes entre as cadeias (ligações cruzadas) fornecem aos PUs termorrígidos propriedades superiores às verificadas para os PUs termoplásticos como resistências à tração, compressão, impacto, abrasão e à degradação causada por ácidos, bases e solventes orgânicos, mantendo as características elastoméricas ${ }^{[4]}$. Esses polímeros são geralmente preparados via processamento de pré-polímero, uma vez que possibilita um melhor controle da reação química antes da etapa de reticulação, empregando-se proporções molares da ordem de 1,00 mol de diol para $1,60-2,25$ mols de isocianato ${ }^{[2,3]}$. Os poliuretanos termorrígidos elastoméricos com excelentes propriedades de resistência ao desgaste são utilizados nas indústrias de mineração, óleo e gás, apresentando grandes vantagens financeiras, sendo utilizados na fabricação de peças estruturais de alto desempenho tais como esteiras de mineração, laminados de interior de aviões ou peças de recepção de grandes tubulações em alto mar (enrijecedores de curvatura $)^{[5,6]}$.

Os nanotubos de carbono (CNTs) são considerados como um dos materiais mais resistentes já sintetizados pelo homem. Apresentam propriedades elétricas e térmicas extraordinárias somadas a uma boa estabilidade química, o que vem despertando grande interesse na sua utilização em diversas aplicações, principalmente no preparo de compósitos poliméricos com elevada resistência mecânica, por exemplo à tração e flexão, assim como grande estabilidade térmic ${ }^{[7,8]}$. Os nanotubos de carbono de paredes múltiplas (MWCNTs) possuem características elétricas entre metal e semicondutor, apresentam módulo de elasticidade de 0,27-0,95 TPa, resistência à tração de 11-63 $\mathrm{GPa}$ e condutividade entre 200-3000 W/mK (MWCNTs isolados) ${ }^{[9-11]}$. A adição de CNTs em matrizes poliméricas permite melhorias nas suas propriedades mecânicas, térmicas, elétricas, entre outras $^{[10-12]}$. A redução no preço dos nanotubos de carbono, especialmente para os MWCNTs, tem contribuído para sua utilização em maiores escalas ${ }^{[13]}$.

Como o processamento dos poliuretanos termorrígidos é completamente distinto, a dispersão de CNTs nessa matriz para o preparo de compósitos exige técnicas de misturas específicas e seu estudo ainda encontra-se em fase incipiente quando comparado às pesquisas com PUs termoplásticos.

Além das características relacionadas ao nanotubo e ao poliuretano discutidas anteriormente, o processo empregado na dispersão dos CNTs é determinante para as propriedades finais do compósito obtido. Os poucos trabalhos voltados para o preparo de nanocompósitos CNTs/PUs termorrígidos encontrados na literatura são baseados na síntese do polímero em duas etapas (via pré-polímero) com a dispersão dos nanotubos no isocianato ou no poliol com o auxílio de dispersores de alto cisalhamento ou ultrassom ${ }^{[14-17]}$. Foram utilizados nanotubos do tipo SWCNTs (nanotubos de carbono de parede simples) e MWCNTs, purificados, modificados e não modificados quimicamente ${ }^{[15-18]}$. Nesses trabalhos, os compósitos foram preparados diretamente na concentração de interesse.

No intuito de contribuir para o tema e acrescentar novos dados ao universo de compósitos com poliuretanos termorrígidos, nosso grupo propôs uma metodologia de preparação de compósito PU termorrígido elastomérico/MWCNT por meio da dispersão dos MWCNTs em pré-polímero empregando dispersor de alto cisalhamento e moinho de rolos a partir de concentrados (masterbatches) que posteriormente foram diluídos para a concentração desejada ${ }^{[1,20]}$. Como continuação desse estudo, o presente trabalho tem como objetivo avaliar um procedimento de dispersão de CNTs por meio da avaliação da morfologia, propriedades térmicas, elétricas e mecânicas dos nanocompósitos obtidos. Foram utilizados MWCNTs não modificados e modificados (com grupos carboxílicos) em poliol por meio de preparação de concentrados $(3,0 \%$ em massa de MWCNTs). Esse trabalho visa contribuir para a introdução de nanotubos de carbono no setor produtivo de poliuretanos termorrígidos buscando diminuir o impacto negativo (financeiro e de saúde, meio ambiente e segurança - SMS) na cadeia de valores do produto final.

\section{Materiais e Métodos}

\subsection{Materiais}

Foram utilizados MWCNTs adquiridos da empresa Timesnano (China) com pureza $>95 \%$, diâmetro externo e comprimento de $2-8 \mathrm{~nm}$ e $1-30 \mu \mathrm{m}$, respectivamente. Dois tipos de nanotubos foram utilizados: não modificado e modificado com grupos carboxilados (4\% de acordo com o fornecedor). Para a síntese do PUE (poliuretano termorrígido elastomérico), foram empregados poli (tetrametileno éter glicol) (PTMG-1000) proveniente da SAFE Chemicals LLC, 2,4-diisocianato de tolueno (TDI) (nome IUPAC 2,4-diisocianato -1-metil benzeno) fornecido pela Bayer S.A., 1,4-butano diol como extensor de cadeia (nome IUPAC butano-1,4-diol) proveniente da M.Cassab Comercio e Indústria LTDA e 4,4-metileno-bis-orto-cloroanilina (MOCA) como agente de cura (nome IUPAC [(4-amino-3-clorofenil) metil]-2-cloroanilina) adquirido da M.Cassab Comercio e Indústria LTDA. Todos os reagentes foram utilizados como recebidos.

\subsection{Métodos}

\subsubsection{Dispersão de MWCNTs em poliol}

Inicialmente os MWCNTs modificados e não modificados foram secos em estufa a vácuo por um período de 10 horas a temperatura de $110^{\circ} \mathrm{C}$ de forma a garantir a ausência de umidade. Posteriormente, a massa desejada de nanotubos (modificados e não modificados) foi dispersa em PTMG-1000 previamente aquecido a $60^{\circ} \mathrm{C}$. Esta mistura originou um concentrado (masterbatch) com teor de 3\% em massa de MWCNTs em Poliol. A dispersão foi realizada através de um misturador de alto cisalhamento (Turrax ${ }^{\circledR}$ ) a uma velocidade de $20000 \mathrm{rpm}$ por $10 \mathrm{~min}$. Após esta etapa a mistura foi processada em moinho de rolos com separação entre os rolos de 10 e $5 \mu \mathrm{m}$ e velocidade de $100 \mathrm{rpm}$ a $60^{\circ} \mathrm{C}$. A Figura 1 mostra as etapas usadas na dispersão.

\subsubsection{Síntese dos nanocompósitos}

Os nanocompósitos foram produzidos em um processo que envolve a síntese de um pré-polímero e sua posterior cura (Figura 1b). A reação para obtenção do pré-polímero foi realizada em um reator de aço inox equipado com agitador mecânico sob vácuo após diluição do concentrado (pela adição de PTMG) para 0,5\% em massa de MWCNTs. Foram adicionadas 


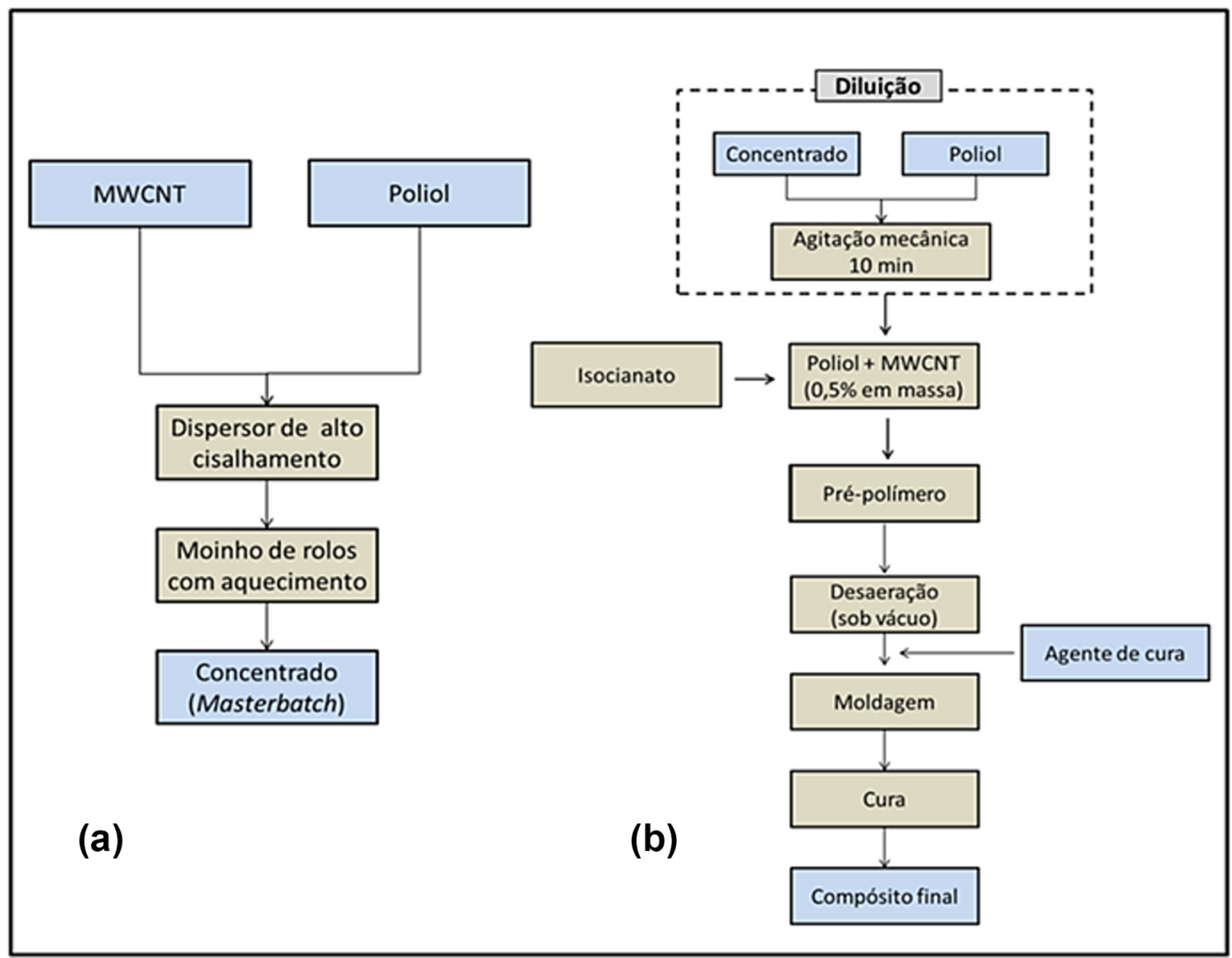

Figura 1. (a) Processo de dispersão dos nanotubos de carbono em poliol em duas etapas, primeiro com o agitador mecânico e posteriormente com o moinho de rolos e (b) produção dos compósitos ${ }^{[21]}$.

quantidades estequiométricas de PTMG 1000/MWCNTs/TDI e o sistema foi mantido a $70^{\circ} \mathrm{C}$ por 2 horas com posterior adição de 1,4-butanodiol com a finalidade de aumentar a massa molar do pré-polímero ${ }^{[22]}$. Após 2 horas de reação, obteve-se o pré-polímero com teor de NCO livre em torno de 7,4\% determinado por titulação com $\mathrm{N}$-dibutilamina ${ }^{[23]}$. A segunda etapa do processo de síntese dos nanocompósitos inicia-se com a desaeração do pré-polímero em câmara de vácuo por $90 \mathrm{~min}$. Antes de adicionar o agente de cura (MOCA) o pré-polímero foi reaquecido a $80^{\circ} \mathrm{C}$. A massa de MOCA utilizada foi proporcional à quantidade de $\mathrm{NCO}$ livre presente no pré-polímero. Após a adição do MOCA, a mistura foi homogeneizada através de uma agitação suave por aproximadamente $1 \mathrm{~min}$ tomando-se o devido cuidado de não inserir bolhas no sistema. Em seguida a mistura foi vertida em moldes que, posteriormente, passaram pelo processo de cura a $100^{\circ} \mathrm{C}$ por $10 \mathrm{~h}$. Posteriormente o processo de pós-cura final foi conduzido mantendo as amostras por 15 dias a temperatura ambiente $e^{[20]}$.

\subsubsection{Caracterização}

A caracterização detalhada das amostras de nanotubos modificados e não modificados foi descrita em um trabalho prévio do nosso grupo ${ }^{[20]}$.
Os nanocompósitos foram caracterizados por microscopia óptica (MO) utilizando um microscópio Olympus, modelo BX50F-e. Sua morfologia foi examinada por microscopia eletrônica de varredura (MEV) utilizando microscópio com canhão de emissão por efeito de campo (Quanta 200 - FEG/FEI). Para esta análise as amostras foram fraturadas em nitrogênio líquido e suas superfícies de fratura foram recobertas por uma fina camada de ouro. Com o propósito de avaliar a dispersão micrométrica e nanométrica dos MWCNTs na matriz de PUE foi utilizada microscopia eletrônica de transmissão (MET) em microscópio Tecnai - G2-20/FEI. Para esta análise, as amostras foram preparadas com o auxílio da técnica de criomicrotomia.

Espectros de absorção no infravermelho do PUE e dos nanocompósitos foram obtidos utilizando espectrômetro FTIR (dispositivo de ATR, modelo 380 Nicolet Thermo Scientific) com uma faixa espectral de $4000-680 \mathrm{~cm}^{-1}$. Foram realizadas 32 varreduras com resolução de $4 \mathrm{~cm}^{-1}$. Medidas elétricas nestas mesmas amostras foram feitas a $25^{\circ} \mathrm{C}$ utilizando um potenciostato Autolab PGSTAT30 Ecochemie. Foi utilizada uma célula experimental de impedancimetria modelo AN8080 da marca Analógica possuindo dois eletrodos bloqueantes de aço inox de área $0,27 \mathrm{~cm}^{2}$. A faixa de frequência usada foi entre $1 \mathrm{MHz}$ e $0,5 \mathrm{~Hz}$, sob $0 \mathrm{~V}$ e amplitude de perturbação 
de $10 \mathrm{mV}$. As amostras foram medidas na forma de filmes com espessura de $0,3 \mathrm{~mm}$.

O PUE e os nanocompósitos foram submetidos a ensaios de tração e medição da dureza (Shore D). Os ensaios de tração foram realizados em máquina de ensaio Kratos modelo TRCV59D-USB com célula de carga de $100 \mathrm{Kgf}$, velocidade de deslocamento de $500 \mathrm{~mm} / \mathrm{min}$ a temperatura de $22,0{ }^{\circ} \mathrm{C}$ e umidade relativa de $44,0 \%$ empregando-se um número mínimo de 03 ensaios para cada amostra. Análises termogravimétricas (TGA) destas amostras foram realizadas em atmosfera de nitrogênio com razão de aquecimento de $10{ }^{\circ} \mathrm{C} / \mathrm{min}$ utilizando o equipamento TGA Q5000 dentro de um intervalo de temperatura de 20 a $800^{\circ} \mathrm{C}$.

\section{Resultados e Discussões}

\subsection{Dispersão dos MWCNTs em poliol}

$\mathrm{Na}$ síntese de poliuretanos empregando-se reação em duas etapas, uma primeira avaliação da dispersão dos nanotubos de carbono pode ser realizada através de imagens obtidas por microscopia ótica. Essa técnica é muito útil para verificar se há presença de agregados de CNTs, inferir sobre o número e tamanho dos mesmos e assim julgar a eficiência do processo de dispersão utilizado ${ }^{[24,25]}$.

A Figura 2 mostra as etapas do processo de dispersão dos nanotubos de carbono obtidas no presente trabalho e as imagens de microscopia óptica do sistema em cada etapa.

Como descrito na literatura, o grande desafio no preparo de nanocompósito poliméricos é a dispersão eficiente dos nanotubos de carbono na matriz, tanto em suspensão quanto no estado sólido ${ }^{[26]}$. A imagem de MO (Figura 2b) mostra a sedimentação dos nanotubos de carbono (MWCNT modificado) após sua adição ao poliol e agitação simples. Para diminuir a viscosidade do meio e melhorar a processabilidade, o sistema foi aquecido a $60{ }^{\circ} \mathrm{C}$. Na primeira etapa, a suspensão foi submetida a um dispersor de alto cisalhamento (Turrax ${ }^{\circledR}$ ) e a melhor dispersão foi obtida após 10 min na velocidade de 20000 rpm. No entanto, constata-se que existem vários agregados de diferentes tamanhos (na faixa de $3 \mu \mathrm{m}$ ate $67 \mu \mathrm{m})$ de nanotubos de carbono não dispersos, o que indica

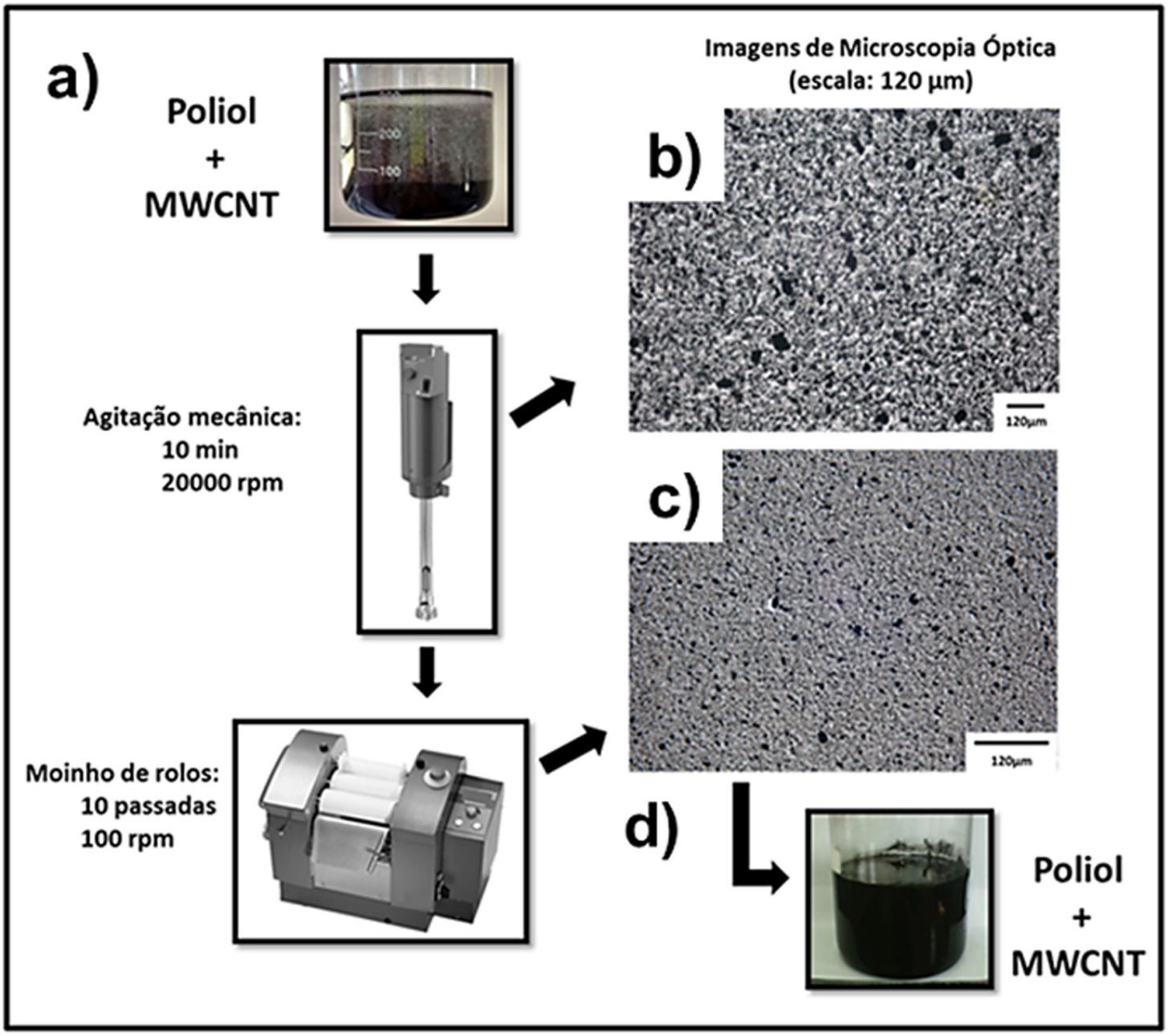

Figura 2. Processo de dispersão dos nanotubos de carbono em poliol em duas etapas: primeiro com o agitador mecânico e posteriormente com o moinho de rolos. (a) Poliol e nanotubo formando duas fases, (b) imagem de MO da dispersão dos CNTs em poliol utilizando apenas agitador mecânico, (c) imagem de MO da dispersão dos CNTs em poliol após as duas etapas de uso do agitador mecânico e do moinho de rolos, (d) CNTs dispersos em poliol formando uma única fase. 
que apenas a utilização do dispersor não é suficiente para atingir o efeito desejado. Na sequência, o sistema foi levado a um moinho de rolos e submetido a diferentes condições de processamento, variando-se a separação entre os rolos, a velocidade de rotação do moinho e o número de vezes pelo qual determinada quantidade de material era submetida aos três rolos ("ciclos"). A imagem de MO (Figura 2c) mostra a dispersão obtida (MWCNT modificado) com separação entre os rolos de 10 e $5 \mu \mathrm{m}$, velocidade de rotação de $100 \mathrm{rpm}$ após 10 ciclos. Embora ainda existam pequenos agregados (entre 0,7 e $21 \mu \mathrm{m}$ ), verifica-se que a quantidade de material disperso aumentou com a introdução da etapa de moagem. O aspecto da dispersão final é mostrado na Figura 2d. Para o MWCNT não modificado, esta também é a melhor condição de dispersão, embora tenham sido observados agregados maiores e em maior número que para o MWCNT modificado. A melhor dispersão dos MWCNTs modificados está relacionada à sua melhor interação com o PTMG, o que previne a reagregação.

A vantagem do processo de dispersão do nanotubo em poliol em relação ao realizado em pré-polímero é que o controle da umidade não precisa ser rigoroso, o que facilita o transporte e armazenamento do produto. Como já mencionado, a proporção isocianato/poliol é crucial para a obtenção de PUE com propriedades desejadas. Após a reação de formação do pré-polímero, a manutenção da quantidade de isocianato livre calculada para permitir a reação com o agente de cura é importante. A presença de vapor de água altera consideravelmente o teor de NCO livre, e, portanto, exerce grande influência nas características do compósito final.

As imagens de MO mostradas na Figura 2 são relativas ao concentrado (masterbatch) contendo 3\% em massa de CNT funcionalizado. Esse concentrado pode ser armazenado por vários dias sem que nenhuma sedimentação ocorra.

Para preparo dos compósitos, o masterbatch pode ser diluído para a concentração desejada pelo emprego de agitação mecânica simples. Isso facilita a introdução dos CNTs na cadeia produtiva de produtos/peças de poliuretanos termorrígidos, uma vez que não há necessidade de utilização de equipamentos específicos, pois os CNTs já estarão devidamente dispersos no concentrado, nem problemas relacionados à SMS, porque não haverá risco de presença de material particulado sólido suspenso no ar no ambiente de produção.

\subsection{Caracterização dos nanocompósitos}

Para o preparo dos nanocompósitos, os concentrados contendo ambos os MWCNTs (modificados e não modificados) foram diluídos em poliol para concentração de 0,5\% em massa e curados. Os materiais foram caracterizados após 15 dias de pós-cura (temperatura ambiente).

A dispersão dos MWCNTs na matriz e a morfologia dos nanocompósitos foram avaliadas por MO, MEV e MET.

Na Figura 3a, b são apresentadas as imagens de MO para os compósitos preparados com MWCNTs modificados e não modificados. Pode-se constatar que não houve mudança substancial na dispersão/distribuição dos nanotubos no estado sólido em relação aquela verificada para as suspensões em PTMG, ou seja, com as etapas de reação para a síntese de pré-polímero e cura não houve reagregação dos nanotubos de carbono. Tal fato é resultante da eficiência do processo empregado na dispersão para ambos os nanotubos. Como nas suspensões, pode-se observar a presença de aglomerados menores de MWCNTs modificados (entre 0,6 e $12 \mu \mathrm{m}$ ) em relação aos MWCNTs não modificados (faixa de $0,8 \mu \mathrm{m}$ ate $27,0 \mu \mathrm{m}$ ), provavelmente devido a sua melhor interação com a matriz de PUE.

As imagens de MEV (Figura 3c, d) corroboram o que foi pressuposto a partir das imagens de MO. Percebe-se que os MWCNTs modificados apresentaram uma melhor adesão à matriz polimérica como resultado da interação MWCNT-COOH:PUE.

Além disso, como destacado por McClory e colaboradores ${ }^{[15]}$, outro indício dessa interação é que as imagens foram obtidas na superfície de fratura e não foram observadas regiões que indicassem que o MWCNT foi puxado para fora da matriz no momento da fratura. De fato, essa interface MWCNT:matriz é essencial para o reforço mecânico no sistema. As imagens das Figura 3c, d exibem MWCNTs curvados e entrelaçados na matriz polimérica, ligando "pontos" da superfície fraturada. Essa morfologia, verificada em outros compósitos nanotubo:PUs (termorrígido ou termoplástico), é essencial para a transferência de tensão entre os CNTs e a matriz quando o material é sujeito a uma solicitação mecânica ${ }^{[15,27]}$. Como evidência do recobrimento pelo polímero, o diâmetro dos nanotubos de carbono visíveis nas imagens é da ordem de $23 \mathrm{~nm}$ a 34,0 nm nos compósitos com MWCNTs não modificados e de $12,0 \mathrm{~nm}$ a $36,0 \mathrm{~nm}$ nos compósitos com MWCNTs modificados. Valores bem menores foram observados para esses nanotubos isolados (diâmetro na faixa de 5,0 nm a 15,0 nm). Além disso, como os MWCNTs possuem apenas $4 \%$ de funcionalização, não deve haver prejuízo considerável às suas propriedades elétricas, e a morfologia verificada também é propícia à obtenção de boas propriedades elétricas para os compósitos.

A melhor dispersão dos MWCNTs modificados também foi evidenciada nas imagens de MET (Figura 3e, f). Para o nanocompósito preparado com MWCNTs não modificados (Figura 3e), observou-se uma baixa distribuição nanométrica destas cargas com presença significativa de agregados. No caso de utilização de MWCNTs modificados (Figura 3f), pode ser verificada uma melhor distribuição dos MWCNTs por toda a região do nanocompósito, uma vez que, além de agregados nanométricos, há a presença de nanotubos individuais dispersos ao longo da matriz ${ }^{[16]}$.

Assim, constata-se que o método de preparo dos nanocompósitos a partir de masterbatch propicia uma boa dispersão dos nanotubos de carbono na matriz de PUE e que os MWCNTs modificados apresentam-se como materiais mais indicados para preparação de compósitos com propriedades melhoradas.

Segundo a literatura ${ }^{[18]}$, com o tratamento ácido são gerados grupos oxigenados quimissorvidos à superfície dos nanotubos de carbono, como os anidridos de ácido, carbonilas, hidroxilas, grupos carboxílicos, fenólicos e lactônicos, sendo esses três últimos os principais. Os grupos fenólicos, carboxílicos e álcoois são de particular interesse uma vez que podem participar da reação de polimerização com o isocianato originando grupos amida e uretano que 

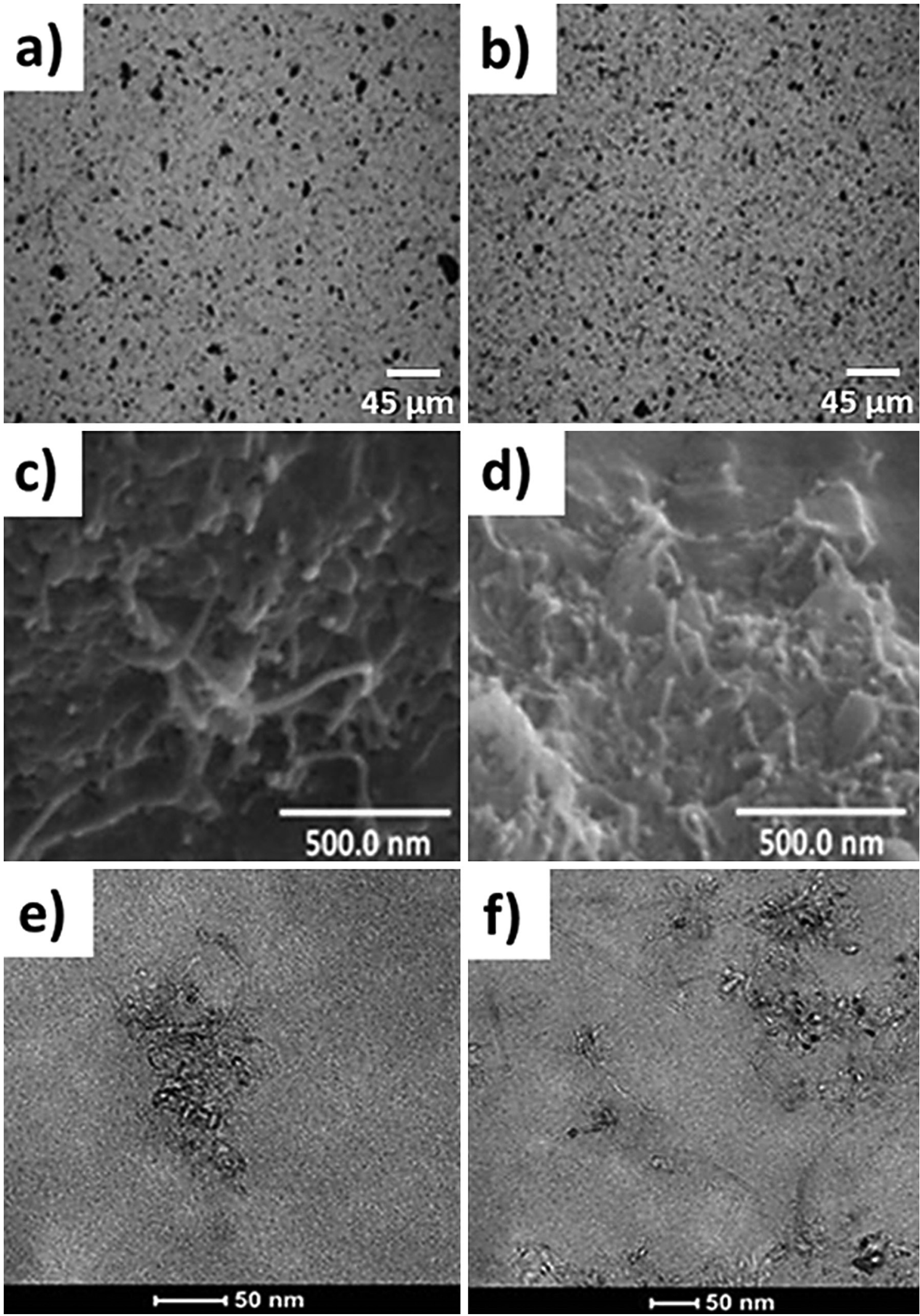

Figura 3. Imagens de MO da superfície dos compósitos contendo 0,5\% em massa de: (a) MWCNTs não modificados e (b) MWCNTs modificados. Imagens de MEV da superfície da fratura dos nanocompósitos contendo $0,5 \%$ em massa de: (c) MWCNT não modificados e (d) MWCNTs modificados e imagens de MET de cortes de criomicrotomia dos nanocompósitos contendo 0,5\% em massa de: (e) MWCNTs não modificados e (f) MWCNTs modificados. 
contribuem para a formação de ligações cruzadas no material final ${ }^{[18]}$. Diante disso, a espectroscopia vibracional na região do infravermelho foi utilizada para estudar os diferentes materiais.

A Figura 4 apresenta o espectro de FTIR do PUE e dos nanocompósitos contendo nanotubos de carbono modificados e não modificados.

Através da análise da Figura 4 pode-se observar uma banda larga de baixa intensidade em $3277 \mathrm{~cm}^{-1}$ característica da própria deformação axial da ligação de hidrogênio do grupo N-H com o oxigênio derivado o grupo éter do segmento flexível ${ }^{[16,28,29]}$. Uma banda na região de $2937 \mathrm{~cm}^{-1}$ corresponde à deformação axial assimétrica de C-H dos

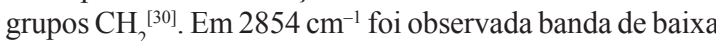
intensidade correspondente à deformação axial de $\mathrm{CH}_{2}$ alifático $^{[30]}$. Em $1695 \mathrm{~cm}^{-1}$ observa-se banda característica de baixa intensidade correspondente à deformação axial $\mathrm{C}=\mathrm{O}$ interagindo com ligação de hidrogênio. As bandas agudas que aparecem em 1525 e $1220 \mathrm{~cm}^{-1}$ podem ser atribuídas às ligações $\mathrm{C}-\mathrm{N}$ de grupos uretanos ${ }^{[30]}$. $\mathrm{A}$ banda em $1101 \mathrm{~cm}^{-1}$ corresponde à deformação axial assimétrica e simétrica da ligação C-O-C $\mathrm{C}^{[30,31]}$. Para os nanocompósitos, não foram observadas bandas adicionais ou deslocamentos consideráveis em relação à PUE, nem diferenças devido à funcionalização dos nanotubos. Possivelmente isso está relacionado à baixa concentração de nanotubos utilizada, bem como à presença de apenas $4 \%$ de grupos carboxílicos no MWCNT modificado. Dessa forma não foi possível avaliar a diferença entre os MWCNTs, nem se há interação preferencial com os domínios rígidos ou flexíveis do poliuretano termorrígido.

A degradação térmica de poliuretanos é um processo complexo e pode ocorrer em pelo menos duas etapas conforme relatado na literatura ${ }^{[32]}$. A primeira etapa está relacionada à decomposição térmica dos segmentos rígidos e a segunda à degradação dos segmentos flexíveis ${ }^{[32-34]}$. As curvas TG e DTG para o PUE e os nanocompósitos são apresentadas na Figura 5. Por meio da curva DTG, pode-se observar que a degradação térmica do PUE acontece em pelo menos três estágios, com máximos em 287, 340, $405^{\circ} \mathrm{C}$.

A decomposição térmica dos nanocompósitos também ocorreu em três estágios, apresentando pequenas variações de temperatura quando comparados ao PUE. Os resultados mostraram que a estabilidade térmica dos nanocompósitos diminuiu em $\sim 12$ e $9{ }^{\circ} \mathrm{C}$ para os nanocompósitos contendo MWCNTs não modificados e modificados, respectivamente. O decréscimo na temperatura de degradação para as amostras de MWCNTs não modificados possivelmente ocorreu devido à dispersão dos mesmos na matriz com presença de maiores agregados, observada pelas imagens de microscopias, e à presença de impurezas adsorvidas nas paredes externas dos tubos que contribuíram para um aumento na velocidade de degradação dos segmentos rígidos deste nanocompósito ${ }^{[29,35]}$. Já para os nanocompósitos contendo MWCNTs modificados, a menor variação provavelmente é devido à melhor dispersão e remoção de impurezas adsorvidas nas superfícies dos tubos como consequência do processo de modificação química.

A fim de avaliar a influência dos MWCNTs nas propriedades mecânicas da matriz de PUE, ensaios de tração e dureza foram realizados. Os resultados obtidos para o PUE

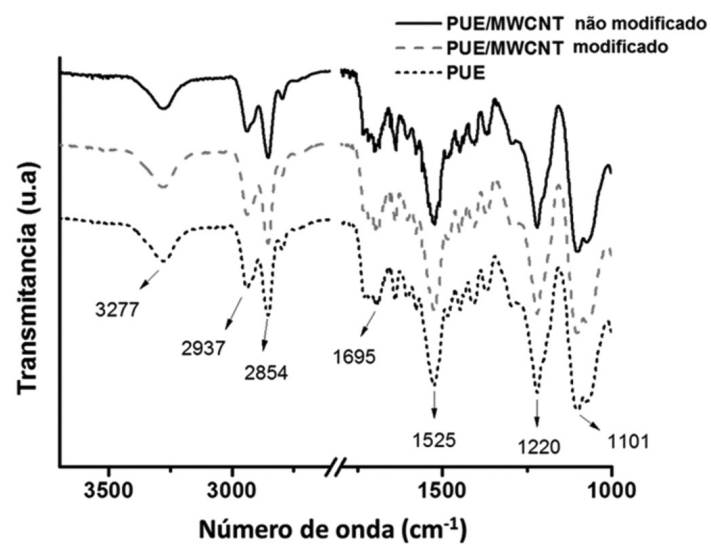

Figura 4. Espectros de FTIR do PUE e dos nanocompósitos contendo $0,5 \%$ em massa de MWCNTs modificados e não modificados.

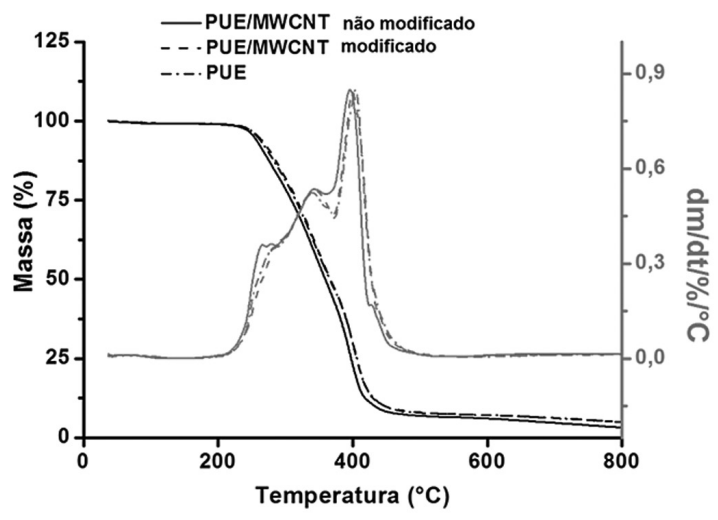

Figura 5. Curva TG e DTG para a PUE e nanocompósitos em atmosfera de $\mathrm{N}_{2}$.

e nanocompósitos produzidos com MWCNTs modificados e não modificados são apresentados na Figura 6

Como já discutido, além das características dos nanotubos de carbono e do procedimento utilizado na dispersão dos mesmos, a natureza da matriz polimérica tem grande influência nas propriedades dos nanocompósitos poliméricos. Portanto, utilizou-se como estratégia para dispersão dos MWCNTs e aumento nas propriedades mecanicas, avaliar a influência do processo pela comparação entre as propriedades dos compósitos preparados com a mesma matriz e nanotubos modificados e não modificados.

A tensão na ruptura reduziu cerca de $7 \%$ com a introdução de MWCNTs não modificados. Já no nanocompósito produzido a partir de MWCNTs modificados verificou-se um aumento em torno de $10 \%$ em relação à matriz de PUE (Figura 6a). O alongamento na ruptura apresentou um comportamento semelhante, com uma redução de cerca de $3 \%$ para o nanocompósito produzido a partir de MWCNTs não modificados e um aumento em torno de $10 \%$ para o nanocompósito com MWCNTs modificados, comparando-se aos valores da PUE (Figura 6b). Para o módulo de elasticidade há uma diminuição de cerca de 17\% 
no nanocompósito preparado com MWCNT não modificado e de aproximadamente $8 \%$ para o nanocompósito com MWCNT modificado (Figura 6c). Através da Figura 6d, pode-se verificar que os nanotubos de carbono exercem pouca influência na dureza (Shore D) da matriz.

Esses resultados corroboram com o que foi observado nos estudos de microscopia, uma vez que mostram que os MWCNTs modificados por tratamento ácido são mais indicados como carga de reforço devido a melhor interação com a matriz e formação de uma interface propícia para a transferência de tensão entre o nanotubo de carbono e o polímero quando o compósito é submetido à tensão mecânica. Esse comportamento foi mostrado na literatura para compósitos preparados com matrizes termorrígidas distintas da utilizada no presente trabalho ${ }^{[18,36]}$.

Para compósitos poliméricos em geral, termoplásticos ou termorrígidos, com MWCNTs, a tendência mais frequente é o aumento no módulo de elasticidade e a diminuição no alongamento de ruptura devido à inserção de uma carga rígida. No entanto, as potenciais influências dos MWCNTs são amplas e também é possível um aumento no alongamento concomitante ao módulo de elasticidade, como já foi relatado para sistemas de PUE/MWCNTs ${ }^{[14]}$, bem como o aumento no alongamento com redução no módulo ${ }^{[37,38]}$. O efeito nas propriedades é específico para cada sistema, considerando tipo de nanotubo e tipo de matriz polimérica. Guo e colaboradores relataram uma diminuição do módulo e um aumento do alongamento para compósitos de epóxi (termorrígido de alto módulo) de forma proporcional à concentração utilizada de MWCNTs funcionalizados ${ }^{[38]}$. Eles discutiram que o aumento na deformação pode ser resultante do deslizamento de camadas internas dos MWCNTs. Portanto, neste caso os nanotubos de carbono atuaram como aditivo para melhorar a ductilidade e não a rigidez do sistema epóxi. Através da funcionalização, esse deslizamento pode ser favorecido devido à camada externa de MWCNTs ser mais defeituosa, a qual interage de forma mais fraca com as camadas internas.

Outro fator importante para as propriedades observadas é que a formulação de PUE estudada apresenta um módulo muito elevado, de $290 \mathrm{MPa}$ (valor obtido pela extrapolação da secante a $2 \%$ de deformação), enquanto os demais trabalhos na literatura sobre PUE/MWCNTs reportam PUE com módulos abaixo de $10 \mathrm{MPa}^{[14,16,17]}$. Assim, apesar dos MWCNTs resultarem em redução no módulo, seu valor ainda é mantido em um patamar elevado com a introdução de MWCNTs funcionalizados.

A introdução de MWCNTs funcionalizados propiciou um aumento da ductilidade do material e o aumento na tensão na ruptura em $10 \%$, o que é o resultado de ganho

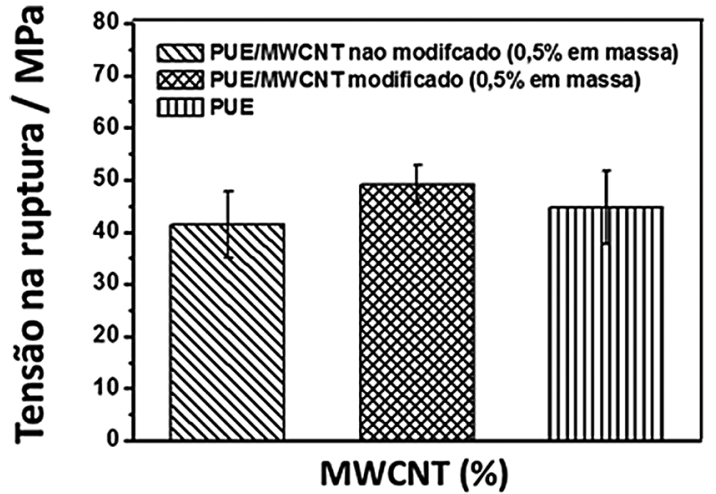

(a)

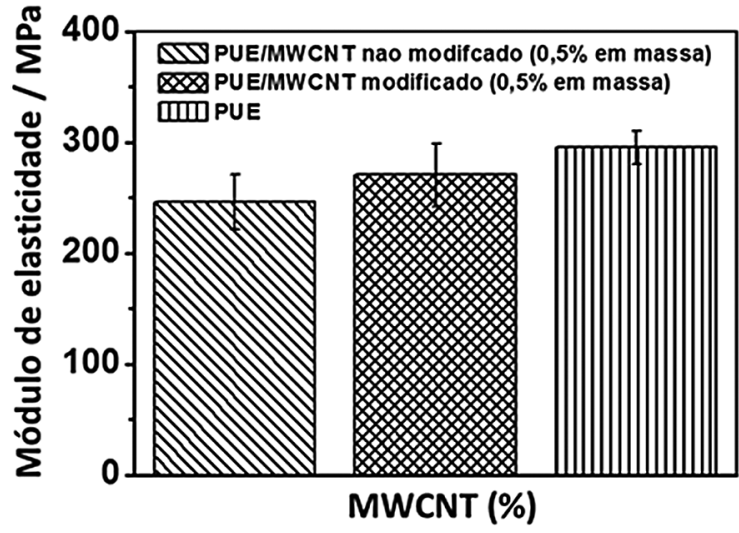

(c)

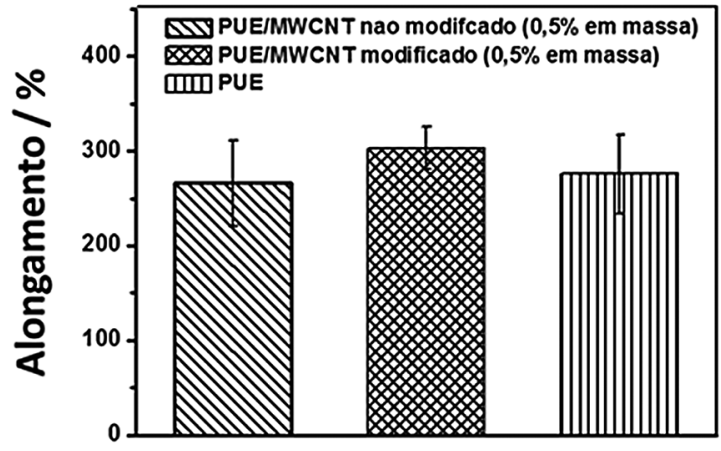

MWCNT (\%)

(b)

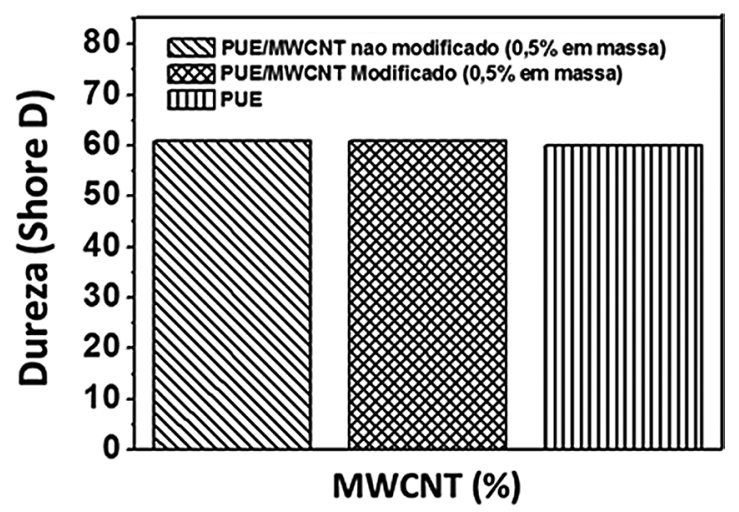

(d)

Figura 6. Propriedades mecânicas das amostras estudas. (a) Tensão na ruptura, (b) alongamento percentual na ruptura, (c) módulo de elasticidade (secante a $2 \%$ ) e (d) dureza (Shore D). 
mais significativo. No caso deste tipo de termorrígido um ganho nesta faixa já é de relevância para considerar-se a aplicação do compósito em projetos de engenharia exigentes. Quando a dispersão de MWCNTs é ineficaz e o compósito é formado por aglomerados grandes, estes atuam como concentradores de tensão, que facilitam a propagação de trincas e reduzem a tensão na ruptura do material ${ }^{[17]}$. Portanto, somente uma nanocarga especial pode promover algum ganho em propriedades mecânicas para polímeros desta classe.

Embora aumentos modestos tenham sido verificados em relação a alguns desses trabalhos, o procedimento descrito permite a preparação de quantidades escaláveis de concentrado. Um diferencial importante para essa rota de processo, que constitui uma maior viabilidade para introdução na indústria, é a utilização de moinho de rolos como equipamento principal de processamento. As referências de PUE/MWCNTs relatam a incorporação da nanocarga por ultrassom ou dispersores de alto cisalhamento ${ }^{[14-16]}$. No entanto, essas técnicas são baseadas em fontes de dispersão localizadas (nos transdutores ultrassônicos ou nas pontas dos dispersores de alto cisalhamento) e, portanto, apresentam dificuldades no aumento de escala sem resultar em heterogeneidades no processamento. $\mathrm{O}$ moinho de rolos garante um processamento homogêneo, onde todo o material é submetido a uma mesma tensão de cisalhamento, sendo uma técnica de alto interesse tecnológico para o aumento de escala. Além disso, os nanotubos de carbono utilizados foram modificados com apenas $4 \%$ de grupos carboxílicos (segundo dados do fornecedor e confirmados por TGA). Nosso grupo desenvolveu uma metodologia de funcionalização por tratamento ácido em escala que possibilita a obtenção de nanotubos de carbono com maiores graus de funcionalização sem variação drástica na sua razão de aspecto e com a utilização de quantidades muito pequenas de ácido ${ }^{[21]}$. Esse procedimento de modificação de superfície dos nanotubos de carbono é muito mais simples dos que as utilizadas nos trabalhos citados anteriormente ${ }^{[37,38]}$. Assim, acredita-se que a utilização de nanotubos com maior grau de funcionalização aliado ao processo de dispersão otimizado no presente trabalho, trarão efeitos mais significativos nas propriedades mecânicas.

A dispersão de nanocargas em polímeros isolantes produz um aumento significativo na condutividade elétrica do material quando a concentração dos nanotubos de carbono atinge o limiar de percolação (aumento de várias ordens de grandeza na condutividade do material em relação à matriz polimérica). Os nanocompósitos obtidos neste trabalho foram estudados por espectroscopia de impedância eletroquímica e a condutividade elétrica foi obtida com o emprego da Equação 127,39].

$$
C=\frac{l}{A \times R}
$$

sendo 1 a espessura, A a área e $\mathrm{R}$ a resistência dos filmes.

A Tabela 1 mostra os resultados de condutividade para todas as amostras investigadas.

Os nanocompósitos com MWCNTs modificados e não modificados apresentaram valores de condutividades superiores em aproximadamente duas ordens de grandeza em relação ao valor obtido para a matriz polimérica pura. No entanto, esses valores ainda são menores que
Tabela 1. Resultados de condutividade para PUE e para os diferentes nanocompósitos preparados.

\begin{tabular}{lc}
\hline \multicolumn{1}{c}{ Amostras } & Condutividade (S/cm) \\
\hline PUE & $3,4 \times 10^{-6}$ \\
PUE/MWCNT não modificado 0,5\% & $9,8 \times 10^{-5}$ \\
em massa & \\
PU/MWCNT modificado 0,5\% em massa & $8,7 \times 10^{-5}$ \\
\hline
\end{tabular}

usualmente obtidos considerando-se a alta condutividade dos nanotubos de carbono isolados ${ }^{[40,41]}$. Segundo Lavall e colaboradores, dependendo da adesão do polímero aos CNTs, há formação de uma camada isolante em torno destas partículas condutoras, diminuindo o contato entre os tubos e, em consequência, as propriedades de condução elétrica ${ }^{[27]}$. A condutividade de nanocompósitos do tipo polímero/CNTs depende intrinsecamente dessa relação polímero/nanotubo e é sempre menor do que a condutividade obtida pelo contato direto entre os tubos ${ }^{[42]}$. No presente trabalho, além desse efeito, acredita-se que a concentração utilizada $(0,5 \%$ de MWCNTs) esteja abaixo do limiar de percolação para esse tipo de sistema. Condutividades entre $10^{-7}$ e $10^{-1} \mathrm{~S} / \mathrm{cm}$ já foram descritos na literatura para sistemas semelhantes, embora a maioria dos valores estejam na ordem de $10^{-4} \mathrm{~S} / \mathrm{cm}^{[42]}$. Em um trabalho anterior do grupo foram obtidos valores próximos a $10^{-5} \mathrm{~S} / \mathrm{cm}$ para a condutividade de um sistema baseado em poliuretano termoplástico (TPU) com $1 \%$ em massa de nanotubos modificados ${ }^{[39]}$, mas neste caso o TPU de partida era altamente isolante, apresentando condutividade de $10^{-12} \mathrm{~S} / \mathrm{cm}$. Valores de condutividade da ordem obtida no presente trabalho permitem que o compósito possa ser empregado em aplicações nas quais a dissipação eletrostática seja um requisito ${ }^{[43]}$. Para atingir condutividades que permitam uso em blindagem eletromagnética deve-se adicionar maior quantidade de nanotubos de carbono, mas será necessário também modificação de estratégia de processamento para produzir dispersões adequadas à percolação elétrica ${ }^{[39,43]}$.

\section{Conclusão}

A produção de masterbatches de MWCNTs em poliol através de uma combinação de misturador de alto cisalhamento e moinho de rolos, possibilitou a fabricação de nanocompósitos com $0,5 \%$ em massa de MWCNTs em PUE após diluição, utilizando somente agitação mecânica, sem prejuízo da dispersão dos nanotubos de carbono. Os MWCNTs modificados com grupos oxigenados resultaram em uma melhor dispersão e adesão dos CNTs à matriz polimérica, que refletiram em melhores resultados mecânicos, como tensão na ruptura e alongamento, além de uma melhor preservação da estabilidade térmica. A condutividade dos nanocompósitos foi aumentada em aproximadamente duas ordens de grandeza, o que possibilita o uso do material em aplicações que demandam dissipação eletrostática. A metodologia utilizada garante um processamento homogêneo, com possibilidade de aumento de escala e alta atratividade tecnológica para inserção na indústria. A avaliação do grau de dispersão dos MWCNTs em PUE pelo método desenvolvido neste trabalho e das propriedades obtidas consequentemente, permitem projetar a fabricação de nanocompósitos com diferentes conjuntos de propriedades através do controle do teor de grupos oxigenados nos MWCNTs, da concentração utilizada de nanocarga e das etapas de processo. 


\section{Agradecimentos}

Os autores agradecem a Petrobras, Conselho Nacional de Desenvolvimento Científico e Tecnológico (CNPq), Fundação de Amparo à Pesquisa do estado de Minas Gerais (FAPEMIG), Plastiprene Ltda, Instituto Nacional de Ciência e Tecnologia em Nanomateriais de Carbono (INCT) e ao Centro de Microscopia da UFMG. Magnovaldo C. Lopes e João Paulo C. Trigueiro agradecem ao CNPq e a CAPES pelas bolsas concedidas.

\section{Referências}

1. Prisacariu, C. (2011). Polyurethane elastomers: from morphology to mechanical aspects. New York: Springer.

2. Dieterich, D., Grigat, E., Hahn, W., Hespe, H., \& Schmelzer, H. G. (1993). Principles of polyurethane chemistry and special applications. 2. ed. Cincinnati: Hanser.

3. Clemitson, I. R.(2008). Castable polyurethane elastomers. New York: CRC Press.

4. Chattopadhyay, D. K., \& Raju, K. V. S. N. (2007). Structural engineering of polyurethane coatings for high performance applications. Progress in Polymer Science, 32(3), 352-418. http://dx.doi.org/10.1016/j.progpolymsci.2006.05.003.

5. Sampurno, Y., Borucki, L., Zhuang, Y., Misra, S., Holland, K., Boning, D., \& Philipossian, A. (2009). Characterization of thermoset and thermoplastic polyurethane pads, and molded and non-optimized machined grooving methods for oxide chemical mechanical planarization applications. Thin Solid Films, 517(5), 1719-1726. http://dx.doi.org/10.1016/j. tsf.2008.09.077.

6. Ledru, Y., Bernhart, G., Piquet, R., Schmidt, F., \& Michel, L. (2010). Coupled visco-mechanical and diffusion void growth modelling during composite curing. Composites Science and Technology, 70(15), 2139-2145. http://dx.doi.org/10.1016/j. compscitech.2010.08.013.

7. Wang, T.-L., \& Tseng, C.-G. (2007). Polymeric carbon nanocomposites from multiwalled carbon nanotubes functionalized with segmented polyurethane. Journal of Applied Polymer Science, 105(3), 1642-1650. http://dx.doi. org/10.1002/app.26224.

8. Wei, B. Q., Vajtai, R., \& Ajayan, P. M. (2001). Reliability and current carrying capacity of carbon nanotubes. Applied Physics Letters, 79(8), 1172-1174. http://dx.doi.org/10.1063/1.1396632.

9. Yang, D. J., Wang, S. G., Zhang, Q., Sellin, P. J., \& Chen, G. (2004). Thermal and electrical transport in multi-walled carbon nanotubes. Physics Letters, Part A, 329(3), 207-213. http://dx.doi.org/10.1016/j.physleta.2004.05.070.

10. Yu, M.-F., Lourie, O., Dyer, M. J., Moloni, K., Kelly, T. F., \& Ruoff, R. S. (2000). Strength and breaking mechanism of multiwalled carbon nanotubes under tensile load. Science, 287(5453), 637-640. http://dx.doi.org/10.1126/science.287.5453.637. PMid:10649994.

11. Moniruzzaman, M., \& Winey, K. I. (2006). Polymer nanocomposites containing carbon nanotubes. Macromolecules, 39(16), 5194-5205. http://dx.doi.org/10.1021/ma060733p.

12. Silva, M. A., Tavares, M. I. B., Nascimento, S. A. M., Rodrigues, E., \& Jd, R. (2012). Caracterização de nanocompósitos de poliuretano/montmorilonita organofílica por RMN de baixo campo. Polímeros: Ciência e Tecnologia, 22(5), 481-485. http://dx.doi.org/10.1590/S0104-14282012005000064.

13. De Volder, M. F. L., Tawfick, S. H., Baughman, R. H., \& Hart, A. J. (2013). Carbon Nanotubes: Present and Future Commercial Applications. Science, 339(6119), 535-539. http:// dx.doi.org/10.1126/science.1222453. PMid:23372006.
14. Kantheti, S., Gaddam, R. R., Narayan, R., \& Raju, K. V. (2014). Hyperbranched polyol decorated carbon nanotube by click chemistry for functional polyurethane urea hybrid composites. RSC Advances, 4(47), 24420-24427. http://dx.doi. org/10.1039/c4ra02442g.

15. McClory, C., McNally, T., Brennan, G. P., \& Erskine, J. (2007). Thermosetting polyurethane multiwalled carbon nanotube composites. Journal of Applied Polymer Science, 105(3), 1003-1011. http://dx.doi.org/10.1002/app.26144.

16. Xiong, J., Zheng, Z., Qin, X., Li, M., Li, H., \& Wang, X. (2006). The thermal and mechanical properties of a polyurethane/ multi-walled carbon nanotube composite. Carbon, 44(13), 2701-2707. http://dx.doi.org/10.1016/j.carbon.2006.04.005.

17. Xia, H., \& Song, M. (2006). Preparation and characterisation of polyurethane grafted single-walled carbon nanotubes and derived polyurethane nanocomposites. Journal of Materials Chemistry, 16(19), 1843-1851. http://dx.doi.org/10.1039/ b601152g.

18. Karabanova, L. V., Whitby, R. L. D., Korobeinyk, A., Bondaruk, O., Salvage, J. P., Lloyd, A. W., \& Mikhalovsky, S. V. (2012). Microstructure changes of polyurethane by inclusion of chemically modified carbon nanotubes at low filler contents. Composites Science and Technology, 72(8), 865-872. http:// dx.doi.org/10.1016/j.compscitech.2012.02.008.

19. Lopes, M. C., Silva, G. G., Lavall, R. L., Diniz, V. P. A., \& Castro, V. G. (2013). BR Patente No 10201330082961. Brasília: Instituto Nacional de Propriedade Industrial.

20. Lopes, M. C., Castro, V.G., Seara, L. M., Diniz, V. P. A., Lavall, R. L., \& Silva, G. G. (2014). Thermosetting polyurethanemultiwalled carbon nanotube composites: thermomechanical properties and nanoindentation. Journal of Applied Polymer Science, 131(23) 41207

21. Silva, G. G., Lavall, R. L., Figueiredo, K. C. S., Castro, V. G., Costa, I. B., Medeiros, F. S., Lopes, M. C., Ferreira, F. L. Q., \& Diniz, V. P. A. (2014). BR Patente No 1020140259660. Brasília: Instituto Nacional de Propriedade Industrial.

22. Delpech, M. C., Coutinho, F. M. B., Sousa, K. G. M., \& Cruz, R. C. (2007). Estudo Viscosimétrico de Prepolímeros Uretânicos. Polimeros: Ciência e Tecnologia, 17(4), 294-298. http://dx.doi.org/10.1590/S0104-14282007000400008.

23. Pacheco, M. F. M., Fiorio, R., Zattera, A. J., Zeni, M., \& Crespo, J. S. (2007). Efeito da concentração de segmentos rígidos nas propriedades físico-mecânicas, químicas e na morfologia de elastômeros microcelulares de poliuretano. Polímeros: Ciência e Tecnologia, 17(3), 234-239. http://dx.doi. org/10.1590/S0104-14282007000300013.

24. Ryszkowska, J. (2009). Quantitative image analysis of polyurethane/carbon nanotube composite microstructures. Materials Characterization, 60(10), 1127-1132. http://dx.doi. org/10.1016/j.matchar.2009.01.021.

25. Ryszkowska, J., Jurczyk-Kowalska, M., Szymborski, T., \& Kurzydlowski, K. J. (2007). Dispersion of carbon nanotubes in polyurethane matrix. Physica E: Low-Dimensional Systems and Nanostructures, 39(1), 124-127. http://dx.doi.org/10.1016/j. physe.2007.02.003.

26. Ma, P.-C., Siddiqui, N. A., Marom, G., \& Kim, J.-K. (2010). Dispersion and functionalization of carbon nanotubes for polymer-based nanocomposites: a review. Composites Part A: Applied Science and Manufacturing, 41(10), 1345-1367. http://dx.doi.org/10.1016/j.compositesa.2010.07.003.

27. Lavall, R. L., Sales, J. A., Borges, R. S., Calado, H. D. R., Machado, J. C., Windmoller, D., Silva, G. G., Lacerda, R. G., \& Ladeira, L. O. (2010). Nanocompósitos de poliuretana termoplástica e nanotubos de carbono de paredes múltiplas para dissipação eletrostática. Quimica Nova, 33(1), 133-140. http://dx.doi.org/10.1590/S0100-40422010000100025. 
28. Wen, T.-C., Du, Y.-L., \& Digar, M. (2002). Compositional effect on the morphology and ionic conductivity of thermoplastic polyurethane based electrolytes. European Polymer Journal, 38(5), 1039-1048. http://dx.doi.org/10.1016/S0014-3057(01)00257-9.

29. Xiong, J., Zheng, Z., Song, W., Zhou, D., \& Wang, X. (2008). Microstructure and properties of polyurethane nanocomposites reinforced with methylene-bis-ortho-chloroanilline-grafted multi-walled carbon nanotubes. Composites. Part A, Applied Science and Manufacturing, 39(5), 904-910. http://dx.doi. org/10.1016/j.compositesa.2007.12.008.

30. Wang, T.-L., Yu, C.-C., Yang, C.-H., Shieh, Y.-T., Tsai, Y.-Z., \& Wang, N.-F. (2011). Preparation, characterization, and properties of polyurethane-grafted multiwalled carbon nanotubes and derived polyurethane nanocomposites. Journal of Nanomaterials, 2011, 1-9. http://dx.doi.org/10.1155/2011/814903. PMid:21808638.

31. Barick, A. K., \& Tripathy, D. K. (2011). Preparation, characterization and properties of acid functionalized multiwalled carbon nanotube reinforced thermoplastic polyurethane nanocomposites. Materials Science and Engineering B, 176(18), 1435-1447. http://dx.doi.org/10.1016/j.mseb.2011.08.001.

32. Chattopadhyay, D. K., \& Webster, D. C. (2009). Thermal stability and flame retardancy of polyurethanes. Progress in Polymer Science, 34(10), 1068-1133. http://dx.doi.org/10.1016/j. progpolymsci.2009.06.002.

33. Hablot, E., Zheng, D., Bouquey, M., \& Avérous, L. (2008). Polyurethanes based on castor oil: kinetics, chemical, mechanical and thermal properties. Macromolecular Materials and Engineering, 293(11), 922-929. http://dx.doi.org/10.1002/ mame. 200800185.

34. Javni, I., Petrović, Z. S., Guo, A., \& Fuller, R. (2000). Thermal stability of polyurethanes based on vegetable oils. Journal of Applied Polymer Science, 77(8), 1723-1734. http:// dx.doi.org/10.1002/1097-4628(20000822)77:8<1723::AIDAPP9>3.0.CO;2-K.

35. Mondal, S., \& Hu, J. L. (2006). Thermal degradation study of functionalized MWNT reinforced segmented polyurethane membrane. Journal of Elastomers and Plastics, 38(3), 261-271. http://dx.doi.org/10.1177/0095244306064237.

36. Karabanova, L., Whitby, R. D., Bershtein, V., Korobeinyk, A., Yakushev, P., Bondaruk, O., Lloyd, A., \& Mikhalovsky,
S. (2013). The role of interfacial chemistry and interactions in the dynamics of thermosetting polyurethane-multiwalled carbon nanotube composites at low filler contents. Colloid \& Polymer Science, 291(3), 573-583. http://dx.doi.org/10.1007/ s00396-012-2745-4.

37. Zhao, C., Ji, L., Liu, H., Hu, G., Zhang, S., Yang, M., \& Yang, Z. (2004). Functionalized carbon nanotubes containing isocyanate groups. Journal of Solid State Chemistry, 177(12), 4394-4398. http://dx.doi.org/10.1016/j.jssc.2004.09.036.

38. Song, H.-J., Zhang, Z.-Z., \& Men, X.-H. (2007). Surfacemodified carbon nanotubes and the effect of their addition on the tribological behavior of a polyurethane coating. European Polymer Journal, 43(10), 4092-4102. http://dx.doi.org/10.1016/j. eurpolymj.2007.07.003.

39. Lima, A. M. F., Castro, V. G. D., Borges, R. S., \& Silva, G. G. (2012). Electrical conductivity and thermal properties of functionalized carbon nanotubes/polyurethane composites. Polimeros Ciência e Tecnologia, 22(2), 117-124. http://dx.doi. org/10.1590/S0104-14282012005000017.

40. Ajayan, P. M. (1999). Nanotubes from carbon. Chemical Reviews, 99(7), 1787-1800. http://dx.doi.org/10.1021/cr970102g. PMid:11849010.

41. Barrau, S., Demont, P., Peigney, A., Laurent, C., \& Lacabanne, C. (2003). DC and AC conductivity of carbon nanotubespolyepoxy composites. Macromolecules, 36(14), 5187-5194. http://dx.doi.org/10.1021/ma021263b.

42. Musumeci, A. W., Silva, G. G., Liu, J.-W., Martens, W. N., \& Waclawik, E. R. (2007). Structure and conductivity of multiwalled carbon nanotube/poly(3-hexylthiophene) composite films. Polymer, 48(6), 1667-1678. http://dx.doi.org/10.1016/j. polymer.2007.01.027.

43. Ramasubramaniam, R., Chen, J., \& Liu, H. (2003). Homogeneous carbon nanotube/polymer composites for electrical applications. Applied Physics Letters, 83(14), 2928-2930. http://dx.doi. org/10.1063/1.1616976.

Enviado: Maio 31, 2015

Revisado: Ago. 13, 2015 Aceito: Set. 02, 2015 